Pacific Journal of Mathematic 


\title{
FINITE-TO-ONE OPEN MAPS OF GENERALIZED METRIC SPACES
}

\author{
RAYMOND F. GiTTINGS
}

\begin{abstract}
We investigate the invariance of certain classes of generalized metric spaces under finite-to-one open maps. In particular, the following classes of spaces are invariant: $w \Delta$ -

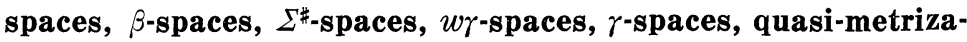
ble $T_{1}$-spaces, $\sigma$-spaces and Moore spaces. Several applications are given, including a metrization theorem via finite-to-one open maps. We also show that $M$-spaces, $w M$-spaces, $w N$ spaces, and $M_{i}$-spaces $(i=1,2,3)$ are not necessarily preserved by finite-to-one open maps. Further, an example is presented which shows that some of those classes of space which are invariant under finite-to-one open maps are not necessarily invariant under compact open maps.
\end{abstract}

1. Introduction. Y. Tanaka [28] showed that several classes of generalized metric spaces are invariant under finite-to-one open maps. In this paper we extend his results to other classes of spaces. A map $f: X \rightarrow Y$ is finite-to-one if, for every $y \in Y$, the set $f^{-1}(y)$ is a finite subset of $X$. All maps are assumed to be continuous and onto. For other pertinent references the reader is referred to ([8], [9], [24]).

In $\S 2$, the investigation of the invariance of many classes of spaces under finite-to-one open maps is undertaken. An interesting consequence of invariance under finite-to-one open maps is a result on point-finite open covers in Corollary 2.4. Also, in $\S 2$, an example is presented which discusses the situation for compact open maps. Further results on finite-to-one open maps, with respect to certain classes of spaces defined by Hodel [13], are presented in $\S 4$.

In $\S 3$, we use the results of $\S 2$ to obtain some interesting mapping theorems of the following sort: Let $f: X \rightarrow Y$ be a finite-to-one open map of a regular $w \Delta$-space $X$ onto a space $Y$ : (1) If $Y$ is a space with a $G_{i}^{*}$-diagonal, then both $X$ and $Y$ are developable; If $Y$ is a collectionwise normal, $\sigma^{\sharp}$-space, then both $X$ and $Y$ are metrizable.

Before proceeding to our theorems, we consider some of the basic terminology needed for this paper.

A space $X$ is called a $w \Delta$-space [5] if there is a sequence $\left\langle\mathscr{U}_{n}\right\rangle$ of open covers of $X$ such that if $x_{n} \in \operatorname{St}\left(x, \mathscr{C}_{n}\right)$, then the sequence $\left\langle x_{n}\right\rangle$ clusters. If there is a sequence $\left\langle\mathscr{U}_{n}\right\rangle$ of open covers of $X$ such that $\left\{\operatorname{St}\left(x, \mathscr{U}_{n}\right): n=1,2, \cdots\right\}$ is an open basis at $x \in X$, then $X$ is said to be a developable space. A regular developable space is called a Moore 
space.

A space $X$ is called a $\beta$-space [14] if, for each $x \in X$, there is a sequence $\left\{g_{n}(x): n=1,2, \cdots\right\}$ of open neighborhoods of $X$ such that if $x \in g_{n}\left(x_{n}\right)$, then the sequence $\left\langle x_{n}\right\rangle$ clusters.

Instead of giving the definitions of $\sigma^{\sharp}$-spaces [27], $\Sigma^{\sharp}$-spaces [20] and quasi-metrizable spaces [29], we present equivalent formulations which are used in the proof of Theorem 2.1. For the actual definitions of these concepts, the reader is referred to the above references.

Lemma 1.1 (Heath). A space $X$ is a $\sigma^{\sharp}$-space if and only if, for each $x \in X$, there is a sequence $\left\{g_{n}(x): n=1,2, \cdots\right\}$ of open neighborhoods of $x$ satisfying

(a) $\bigcap_{n=1}^{\infty} g_{n}(x)=\{x\}$ for all $x \in X$.

(b) If $y \in g_{n}(x)$, then $g_{n}(y) \subset g_{n}(x)$ for all $x, y \in X$ and $n \in N$.

Lemma 1.2 (Nagata [23]; see also [2]). A space $X$ is a $\Sigma^{\sharp}$-space if and only if, for each $x \in X$, there is a sequence $\left\{g_{n}(x): n=1,2, \cdots\right\}$ of open neighborhoods of $x$ satisfying:

(a) If $y \in g_{n}(x)$, then $g_{n}(y) \subset g_{n}(x)$ for all $x, y \in X$ and $n \in N$.

(b) If $x \in g_{n}\left(x_{n}\right)$, then the sequence $\left\langle x_{n}\right\rangle$ clusters.

Lemma 1.3 (Ribeiro [25]). A $T_{1}$-space $X$ is quasi-metrizable if and only if, for each $x \in X$, there is an open basis $\left\{g_{n}(x): n=1,2, \cdots\right\}$ such that if $y \in g_{n}(x)$, then $g_{n}(y) \subset g_{n-1}(x)$ for all $x, y \in X$ and $n \geqq 2$.

Unless otherwise stated no separation axioms are assumed; however, regular spaces are always assumed to be $T_{1}$ and paracompact means paracompact $T_{2}$. The set of positive integers will be denoted by $N$.

\section{Basic results on finite-to-one open maps.}

THEOREM 2.1. Let $f: X \rightarrow Y$ be a finite-to-one open map.

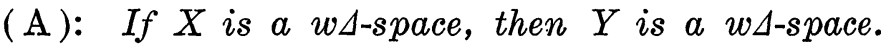

(B): If $X$ is a $\beta$-space, then $Y$ is a $\beta$-space.

(C): If $X$ is a $\sigma^{\sharp}$-space, then $Y$ is a $\sigma^{\sharp}$-space.

(D): If $X$ is a $\Sigma^{\#}$-space, then $Y$ is a $\Sigma^{\#}$-space.

(E): If $X$ is a quasi-metrizable $T_{1}$-space, then $Y$ is quasimetrizable $T_{1}$-space.

Proof. (A ): Suppose $\left\langle\mathscr{U}_{n}\right\rangle$ is a sequence of open covers of $X$ illustrating that $X$ is a $w \Delta$-space. We may assume $\mathscr{U}_{n+1}<\mathscr{U}_{n}$ for each $n \in N$. Let $\mathscr{V}_{n}=f\left(\mathscr{U}_{n}\right)=\left\{f(U): U \in \mathscr{U}_{n}\right\}$ for each $n \in N$. Since $f$ is an open map, $\left\{\mathscr{V}_{n}\right\}$ is a sequence of open covers of $Y$. Suppose 
$y \in Y$ and $y_{n} \in \operatorname{St}\left(y, \mathscr{V}_{n}\right)$ for each $n \in N$. Then, for each $n$, there is an element $V_{n} \in \mathscr{V}_{n}$ such that $\left\{y, y_{n}\right\} \subset V_{n}=f\left(U_{n}\right)$ for some $U_{n} \in \mathscr{U}_{n}$. Choose $x_{n} \in U_{n}$ such that $f\left(x_{n}\right)=y$. Since $f$ is a finite-to-one map, there is an $x \in f^{-1}(y)$ and a subsequence $\left\langle x_{n_{i}}\right\rangle_{i=1}^{\infty}$ of $\left\langle x_{n}\right\rangle$ such that $x_{n_{i}}=x$ for all $i \in N$. Since $y_{n_{i}} \in f\left(U_{n_{i}}\right)$, there is a $z_{n_{i}} \in U_{n_{i}}$ such that $f\left(z_{n_{i}}\right)=y_{n_{i}}$. But then, $\left\{x, z_{n_{i}}\right\} \subset U_{n_{i}}$ and thus $z_{n_{i}} \in \operatorname{St}\left(x, \mathscr{U}_{n_{i}}\right)$. Hence the sequence $\left\langle z_{n_{i}}\right\rangle$ clusters and it follows that the sequence $\left\langle y_{n_{i}}\right\rangle=$ $\left\langle f\left(z_{n_{i}}\right)\right\rangle$ clusters. Thus $Y$ is a $w \Delta$-space.

(B ): For each $x \in X$, let $\left\{g_{n}(x): n=1,2, \cdots\right\}$ be a sequence of open neighborhoods of $x$ illustrating that $X$ is a $\beta$-space. We may assume $g_{n+1}(x) \subset g_{n}(x)$ for each $n \in N$. For each $y \in Y$, let $h_{n}(y)=$ $\bigcap\left\{f\left(g_{n}(x)\right): x \in f^{-1}(y)\right\}$. Since $f$ is an open map, $\left\{h_{n}(y): n=1,2, \cdots\right\}$ is a sequence of open neighborhoods of $y$. Suppose $y \in h_{n}\left(y_{n}\right)$. Then, for each $n$, there is a $z_{n} \in f^{-1}\left(y_{n}\right)$ such that $y \in f\left(g_{n}\left(z_{n}\right)\right)$ and thus an $x_{n} \in g_{n}\left(z_{n}\right)$ such that $f\left(x_{n}\right)=y$. Since $f$ is a finite-to-one map, there is an $x \in f^{-1}(y)$ and a subsequence $\left\langle x_{n_{i}}\right\rangle_{i=1}^{\infty}$ of $\left\langle x_{n}\right\rangle$ such that $x_{n_{i}}=x$ for all $i \in N$. Thus $x \in g_{n_{i}}\left(z_{n_{i}}\right) \subset g_{n}\left(z_{n_{i}}\right)$ and the subsequence $\left\{z_{n_{i}}\right\}_{i=1}^{\infty}$ has a cluster point. Therefore $\left\langle z_{n}\right\rangle$ has a cluster point and thus so does $\left\langle f\left(z_{n}\right)\right\rangle=\left\langle y_{n}\right\rangle$ showing that $Y$ is a $\beta$-space.

$(\mathrm{C})$ : Since $X$ is a $\sigma^{\sharp}$-space, there is a sequence $\left\{g_{n}(x): n=1,2, \cdots\right\}$ of open neighborhoods of each $x \in X$ satisfying conditions (a) and (b) of Lemma 1.1. For each $y \in Y$, let $h_{n}(y)=\bigcap\left\{f\left(g_{n}(x)\right): x \in f^{-1}(y)\right\}$. Since $f$ is an open map, $\left\{h_{n}(y): n=1,2, \cdots\right\}$ is a sequence of open neighborhoods of $y$. To verify condition (1) of Lemma 1.1, suppose $y \in Y$. If $z \in Y$ and $z \neq y$, then $f^{-1}(z) \cap f^{-1}(y)=\varnothing$. Since $f^{-1}(z)$ and $f^{-1}(y)$ are finite sets, there is an integer $n$ such that $g_{n}(x) \cap f^{-1}(z)=\varnothing$ for all $x \in f^{-1}(y)$. It follows that $z \notin \bigcap\left\{f\left(g_{n}(x)\right): x \in f^{-1}(y)\right\}=h_{n}(y)$. Thus $\{y\}=\bigcap_{n=1}^{\infty} h_{n}(y)$. For condition (2) of Lemma 1.1, we suppose $z \in h_{n}(y)$. Then $z \in f\left(g_{n}(x)\right)$ for every $x \in f^{-1}(y)$ and thus there is an $x_{z} \in g_{n}(x)$ such that $f\left(x_{z}\right)=z$. Since $x_{z} \in g_{n}(x), g_{n}\left(x_{z}\right) \subset g_{n}(x)$ and thus $f\left(g_{n}\left(x_{z}\right)\right) \subset f\left(g_{n}(x)\right)$. Thus $\bigcap\left\{f\left(g_{n}\left(x_{z}\right)\right): x \in f^{-1}(y)\right\} \subset \bigcap\left\{f\left(g_{n}(x): x \in f^{-1}(y)\right\}\right.$. It follows that $h_{n}(z) \subset h_{n}(y)$ and thus $Y$ is a $\sigma^{\sharp}$-space.

(D): Using the characterization of $\Sigma^{\#}$-spaces given in Lemma 1.2 , (D) is proved by essentially combining the arguments presented in proving $(\mathrm{B})$ and $(\mathrm{C})$.

(E): By Lemma 1.3, for each $x \in X$, there exists an open basis $\left\{g_{n}(x): n=1,2, \cdots\right\}$ such that if $y \in g_{n}(x)$, then $g_{n}(y) \subset g_{n-1}(x)$ for every $x, y \in X$ and $n \geqq 2$. For each $y \in Y$, let $h_{n}(y)=\bigcap\left\{f\left(g_{n}(x)\right): x \in f^{-1}(y)\right\}$. It is easy to verify that $\left\{h_{n}(y): n=1,2, \cdots\right\}$ is an open basis for $y$. Also, using an argument analogous to that used in (C), it follows that if $z \in h_{n}(y)$, then $h_{n}(z) \subset h_{n-1}(y)$. Thus, since $Y$ is clearly a $T_{1}$-space, $Y$ is quasi-metrizable by Lemma 1.3 .

As immediate consequences of Theorem 2.1, we have the follow- 
ing results concerning $\sigma$-spaces and Moore spaces. As in [28], we define a $\sigma$-space to be a space with a $\sigma$-locally-finite closed net.

Corollary 2.2 (Tanaka [28]). Let $f: X \rightarrow Y$ be a finite-to-one open map of a $\sigma$-space $X$ onto a space $Y$. Then $Y$ is a $\sigma$-space.

Proof. Since $X$ is a $\sigma$-space, $X$ is clearly both a $\Sigma^{\sharp}$ and $\sigma^{\sharp}$-space. Thus, by Theorem 2.1, $Y$ is a $\Sigma^{\sharp}$ and $\sigma^{\sharp}$-space. But a $\Sigma^{\sharp}$ and $\sigma^{\sharp}$-space is a $\sigma$-space [26].

CoRollary 2.3. Let $f: X \rightarrow Y$ be a finite-to-one open map of a Moore space $X$ onto a space $Y$. Then $Y$ is a Moore space.

Proof. Since $X$ is a Moore space, $X$ is a $w \Delta$ and $\sigma^{\sharp}$-space. Thus, by Theorem 2.1, $Y$ is a $w \Delta$ and $\sigma^{\sharp}$-space. Also, since $f$ is a finiteto-one open map and $X$ is regular, it follows that $Y$ is regular. But a regular, $w \Delta$ and $\sigma^{\sharp}$-space is a Moore space [6].

As an immediate consequence of the results of this section we have the following:

CorollaRY 2.4. Let $\left\{0_{\alpha}: \alpha \in A\right\}$ be a point-finite open covering of $X$. If each $0_{\alpha}$ is a w $w$-space ( $\beta$-space; $\sigma^{\sharp}$-space; $\Sigma^{\sharp}$-space, quasimetrizable $T_{1}$-space; $\sigma$-space; Moore space), then so is $X$.

The following example shows that Corollary 2.4 and thus Theorem 2.1 do not hold for $M$-spaces [21], $M^{\sharp}$-spaces [27], $M^{*}$-spaces [15], $w M$-spaces [16] or $M_{i}$-spaces $(i=1,2,3)$ [7].

EXAMPLE 2.5. In [10] Corson and Michael constructed a nonnormal, completely regular space $X$ which is the union of two open metrizable subsets. By Corollary 2.4, $X$ is developable. It follows that $X$ is not an $M, M^{*}, M^{\sharp}, w M$ or $M_{i}$-space $(i=1,2,3)$.

The following example shows that we can not replace the finiteto-one open map by a compact open map in Theorem 2.1 (A), (B), (D) and Corollaries 2.2 and 2.3. This example also shows that semimetrizable and semi-stratifiable spaces [11] are not preserved by compact open maps.

EXAMPLE 2.6. Let $\mathrm{Q}$ be an uncountable subset of $[0,1]$ whose only compact sets are countable, such spaces exist $[17, \mathrm{p} .514]$. Let $Y$ be $[0,1]$ retopologized so that the open sets have the form $U \cup \mathrm{T}^{-}$ where $U$ is open in the usual topology of $[0,1]$ and $V \subset Q$. The space $Y$ is a nonmetrizable, paracompact space with a $G_{\delta}$-diagonal. It [19] Michael showed that the product of $Y$ with a metric space 
is not normal. It follows from [18, Proposition 5] that there is a closed subset of $Y$ which is not a $G_{i}$-set. Thus $Y$ is not semistratifiable and hence not a semimetrizable space, $\sigma$-space, nor a developable space. Further, since $Y$ is a paracompact space with a $G_{i}$-diagonal, $Y$ is not a $\beta$-space nor a $\Sigma^{\sharp}$-space. In [3] Bennett showed that there is a $T_{2}$, metacompact developable space $X$ and a compact open map from $X$ onto $Y$.

We remark that Tanaka [28] constructs many other interesting examples concerning finite-to-one open maps and compact open maps.

3. Applications. A space $X$ is said to have a $G_{\delta}^{*}$-diagonal [14] if there exists a sequence $\left\langle\mathscr{G}_{n}\right\rangle$ of open covers of $X$ such that, for each $x \in X, \bigcap_{n=1}^{\infty} \overline{\operatorname{St}\left(x, \mathscr{G}_{n}\right)}=\{x\}$. According to Hodel [14], a space is a Moore space if and only if it is a regular, $w \Delta$-space witn a $G_{\delta}^{*}$ diagonal

Theorem 3.1. Let $f: X \rightarrow Y$ be a finite-to-one open map of a regular wL-space onto a space $Y$. If $Y$ has a $G_{i}^{*}$-diagonal or is a $\sigma^{\sharp}$-space, then both $X$ and $Y$ are Moore spaces.

Proof. By Theorem 2.1, $Y$ is a $w \Delta$-space. If $Y$ has a $G_{i}^{*}$ diagonal, then $Y$ is a Moore space by Hodel's theorem. If $Y$ is a $\sigma^{\sharp}$-space, then $Y$ is a Moore space by a result of Burke [6, Theorem 2.4]. Thus, in either case, $Y$ is a Moore space. On the other hand, using a result of Tanaka [28] (see also Coban [9]), it follows that $X$ is a $\sigma$-space since $Y$ is a Moore space. But, a $\sigma$-space which is a $w \Delta$-space is a Moore space. Thus $X$ is a Moore space.

Let $\mathscr{U}$ be a collection of open subsets of a space $X$. The collection $\mathscr{U}$ is called point-countable if each point $x \in X$ belongs to at most countable many members of $\mathscr{U}$. The collection $\mathscr{U}$ is called point-separating provided that if $p \neq q$ are points in $X$, there is some $U \in \mathscr{Q}$ such that $p \in U$ and $q \notin U$. Nagata [22] proved that a space is metrizable if and only if it is a paracompact, $w \Delta$-space space with a point-countable, point-separating open cover.

THEOREM 3.2. Let $f: X \rightarrow Y$ be an open finite-to-one map of a regular wL-space $X$ onto a space $Y$. If $Y$ has any one of the following properties, then both $X$ and $Y$ are metrizable.

(a) $Y$ is a paracompact space with a point-countable, pointseparating open cover.

(b) $Y$ is a paracompact space with a $G_{i}$-diagonal.

(c) $Y$ is a collectionwise normal, $\sigma^{*}$-space.

Proof. By Theorem 2.1, $Y$ is a $w \Delta$-space. Thus, in case (a), 
$Y$ is a paracompact, $w \Delta$-space with a point-countable, point-separating open cover and therefore metrizable by Nagata's result. For (b), we note that it is easy to verify that a paracompact space with a $G_{\delta^{-}}$ diagonal has a point-countable, point-separating open cover. Thus (b) follows from (a). For (c), we note that since $Y$ is clearly a regular space, $Y$ is a Moore space by Theorem 3.1. Consequently, since $Y$ is a collectionwise normal Moore space, $Y$ is metrizable [4]. The above arguments show that if $Y$ satisfies (a), (b) or (c), then $Y$ is metrizable. It remains to show that $X$ is metrizable.

To verify the metrizability of $X$ we use the fact that, since $Y$ is metrizable, $Y$ is a hereditarily paracompact, $\sigma$-space. Since $Y$ is hereditarily paracompact and $f$ is a finite-to-one open map, $X$ is hereditarily paracompact [8, Theorem 3]. Furthermore, since $Y$ is a $\sigma$-space and $f$ is a finite-to-one open map, $X$ is a $\sigma$-space ([9], [28]). However, it is well known that a paracompact, $\sigma, w \Delta$-space is metrizable. Thus $X$ is metrizable.

In order to prove Theorems 3.3 and 3.4 we need the following results:

Lemma A (Shiraki [26, Corallary 2.2]). A space $X$ is a $\sigma$-space if and only if $X$ is a $\Sigma^{\sharp}$ and $\sigma^{\sharp}$-space.

Lemma B (Hodel [14]). A $T_{2}$-space $X$ is semi-stratifiable if and only if $X$ is a $\beta$ and $\sigma^{\sharp}$-space.

TheOREM 3.3. Let $f: X \rightarrow Y$ be a finite-to-one open map of a $\sigma^{\sharp}$-space $X$ onto a $\Sigma^{\sharp}$-space $Y$. Then both $X$ and $Y$ are $\sigma$-spaces. Moreover, we may interchange the roles of $X$ and $Y$.

Proof. By Theorem 2.1, $Y$ is a $\sigma^{\sharp}$-space and thus a $\sigma$-space by Lemma A. Since $Y$ is a $\sigma$-space and $f$ a finite-to-one open map $X$ is a $\sigma$-space ([9], [28]). The "moreover" is proved in exactly the same manner.

THEOREM 3.4. Let $f: X \rightarrow Y$ be finite-to-one open map of a $T_{2}$, $\sigma^{*}$-space onto a $\beta$-space $Y$. Then both $X$ and $Y$ are semi-stratifiable. Moreover, we may interchange the roles of $X$ and $Y$.

Proof. By Theorem 2.1, $Y$ is a $\sigma^{\sharp}$-space and thus a semi-stratifiable space by Lemma B. Since $Y$ is semi-stratifiable, $X$ is semistratifiable [28, Theorem 2]. The "moreover" is proved in exactly the same manner.

4. Hodel's spaces. The author is very grateful to the referee 
(R. E. Hodel) for suggestions which led to this portion of the paper.

By Hodel's spaces we mean those classes of spaces which were introduced in [13]; these include $w \gamma$-spaces, $w \theta$-spaces and $w \mathrm{~N}$-spaces.

A space $X$ is a $w \gamma$-space if, for each $x \in X$, there is a sequence $\left\{g_{n}(x): n=1,2, \cdots\right\}$ of open neighborhoods of $x$ such that if $y_{n} \in g_{n}(x)$ and $x_{n} \in g_{n}\left(y_{n}\right)$, then the sequence $\left\langle x_{n}\right\rangle$ has a cluster point. If we require that $x$ is a cluster point of the sequence $\left\langle x_{n}\right\rangle$, then $X$ is called $\gamma$-space [13].

For $w \gamma$-spaces and $\gamma$-spaces we have the following results analogous to Theorem 2.1

THEOREM 4.1. Let $f: X \rightarrow Y$ be a finite-to-one open map.

(a) If $X$ is a wr-space, then $Y$ is a wr-space.

(b) If $X$ is a $\gamma$-space, then $Y$ is a $\gamma$-space.

Proof. (A ): For each $x \in X$, let $\left\{g_{n}(x): n=1,2, \cdots\right\}$ be a sequence of open neighborhoods of $x$ illustrating that $X$ is a $w \gamma$-space. We may assume $g_{n+1}(x) \subset g_{n}(x)$ for each $n \in N$. For each $y \in Y$, let $h_{n}(y)=\bigcap\left\{f\left(g_{n}(x)\right): x \in f^{-1}(y)\right\}$. Since $f$ is an open map, $\left\{h_{n}(y): n \in N\right\}$ is a sequence of open neighborhoods of $y$. Suppose, for $y \in Y$, that $t_{n} \in h_{n}(y)$ and $s_{n} \in h_{n}\left(t_{n}\right)$. Since $f$ is a finite-to-one map and $t_{n} \in h_{n}(y)$, there is a subsequence $\left\langle t_{n_{i}}\right\rangle_{i=1}^{\infty}$ of $\left\langle t_{n}\right\rangle$ such that $t_{n_{i}} \in f\left(g_{n_{i}}(x)\right)$ for some $x \in f^{-1}(y)$. Thus, there is an $x_{n_{i}} \in g_{n_{i}}(x)$ such that $f\left(x_{n_{i}}\right)=t_{n_{i}}$. Also, since $s_{n_{i}} \in h_{n_{i}}\left(t_{n_{i}}\right) \subset f\left(g_{n_{i}}\left(x_{n_{i}}\right)\right)$, there is a $z_{n_{i}} \in g_{n_{i}}\left(x_{n_{i}}\right)$ such that $f\left(z_{n_{i}}\right)=$ $s_{n_{i}}$. Since $x_{n_{i}} \in g_{n_{i}}(x)$ and $z_{n_{i}} \in g_{n_{i}}\left(x_{n_{i}}\right)$, it follows that $\left\langle z_{n_{i}}\right\rangle_{i=1}^{\infty}$ has a cluster point. Therefore, $\left\langle s_{n_{i}}\right\rangle_{i=1}^{\infty}=\left\langle f\left(z_{n_{i}}\right)\right\rangle_{i=1}^{\infty}$ has a cluster point and thus so does $\left\langle s_{n}\right\rangle$ showing that $Y$ is a $w \gamma$-space.

To prove (B), we merely have to observe that if $x$ were a cluster point of $\left\langle z_{n_{i}}\right\rangle_{i=1}^{\infty}$ in the preceding argument, then $y=f(x)$ would be a cluster point of $\left\langle s_{n_{i}}\right\rangle_{i=1}^{\infty}$.

The author has not been successful in obtaining an analogue of the preceding result for $w \theta$-spaces and $\theta$-spaces, although a straight forward argument for these cases yields the following.

THEOREM 4.2. Let $\left\{O_{\alpha}: \alpha \in A\right\}$ be a point-finite open covering of $X$. If each $O_{\alpha}$ is a $\gamma$-space ( $w \gamma$-space; $w \theta$-space; $\theta$-space), then so is $X$.

Turning our attention to $w N$-spaces, we note the following result of Hodel [13]: A space $X$ is a $w M$-space if and only if it is $w N$ space and a $w \gamma$-space. As an immediate consequence of this result and Theorem 4.2, Example 2.5 shows that $w N$-spaces are not preserved by finite-to-one open maps and that Theorem 4.2 does not hold for $w N$-spaces. 
5. Summary. In this section we summarize the known results concerning the invariance of certain classes of generalized metric spaces under finite-to-one open maps.

(A ): Classes of spaces invariant under finite-to-one open maps: In this paper we have shown that $w \Delta$-spaces, $w \gamma$-spaces, $\gamma$-spaces, $\beta$-spaces, $\quad \sigma^{\sharp}$-spaces, $\quad \Sigma^{\sharp}$-spaces, quasi-metrizable $T_{1}$-spaces, $\sigma$-spaces (see also [28]), and Moore spaces are invariant. Also, according to Tanaka [28], $P$-spaces, strict $p$-spaces, symmetric spaces, semimetrizable spaces and semi-stratifiable spaces are invariant. In fact, Henry [12], showed that semi-stratifiable spaces are invariant under pseudoopen finite-to-one maps.

(B): Classes of spaces not invariant under finite-to-one open maps: We have shown in Example 2.5 that $M$-spaces, $w N$-spaces $M^{*}$-spaces, $M^{\sharp}$-spaces, $w M$-spaces and $M_{i}$-spaces $(i=1,2,3)$ are not invariant. Also, Example 3.3 of [28] shows that $\Sigma$-spaces and subparacompact spaces are not invariant.

Finally, we note that it does not seem to be known if $p$-spaces [1] or quasi-complete spaces [11] are preserved by finite-to-one open maps. As mentioned previously, the invariance of $w \theta$-spaces and $\theta$-spaces is not known.

\section{REFERENCES}

1. A. V. Arhangel'skii, On a class of spaces containing all metric spaces and all locally bicompact spaces, Soviet Math. Doki., 4 (1963), 1051-1055.

2. J. M. Atkins, A characterization of $\Sigma$ and $M^{*}$-spaces, Notices Amer. Math. Soc., 18 (1971), 71T-G206.

3. H. R. Bennett, On Arhangel'skii's class MOBI, Proc. Amer. Math. Soc., 26 (1970), 178-180.

4. R. H. Bing, Metrization of topological spaces, Canad. J. Math., 3 (1951), 175-186.

5. C. J. R. Borges, On metrizability of topological spaces, Canad. J. Math., 20 (1968), 795-804.

6. D. K. Burke, On p-spaces and wd-spaces, Pacific J. Math., 35 (1970), 285-296.

7. J.G. Ceder, Some generalizations of metric spaces, Pacific J. Math., 11 (1961), 105-126.

8. M. M. Coban, Open finite-to-one mappings, Soviet Math. Dokl., 8 (1967), 603-605.

9. - On o-paracompact spaces, Vestnik Moskov. Univ., 24 (1), (1969) $20-27=$ Moscow Univ. Bull, 24 (1), (1969), 11-14.

10. H. H. Corson and E. Michael, Metrizability of certain countable unions, Illinois J. Math., 8 (1964), 351-360.

11. G. D. Creede, Concerning semi-stratifiable spaces, Pacific J. Math., 32 (1970), 47-54. 12. M. Henry, Stratifiable spaces, semi-stratifiable spaces, and their relation through mappings, Pacific J. Math., 37 (1971), 697-700.

13. R. E. Hodel, Spaces defined by sequences of open covers which quarantee that certain sequences have cluster points, Duke Math. J., 39 (1972), 253-263.

14. — Moore spaces and w4-spaces, Pacific J. Math., 38 (1971), 641-652. 
15. T. Ishii, On closed mappings and M-spaces I, Proc. Japan Acad., 43 (1967), 752756.

16. - On wM-spaces, I, II, Proc. Japan Acad., 46 (1970), 5-15.

17. K. Kuratowski, Topology, Vol. I, Academic Press, New York and PWN, Warsaw, 1966.

18. E. Michael, A note on paracompact spaces, Proc. Amer. Math. Soc., 4 (1953), 831-835.

19. - The product of a normal space and a metric space need not be normal, Bull. Amer. Math. Soc., 69 (1963), 375-376.

20. On Nagami's $\Sigma$-spaces and some related matters, Proceedings of the Washington State University Conference on General Topology (1970), 13-19.

21. K. Morita, Products of normal spaces with metric spaces, Math. Ann., 154 (1964), 365-382.

22. J. Nagata, A note on Filipov's theorem, Proc. Japan Acad., 45 (1969), 30-33.

23. Characterizations of some generalized metric spaces, Notices Amer. Math. Soc., 18 (1971), 71T-G151.

24. A. Okuyama, Note on inverse images under open finite-to-one mappings, Proc. Japan Acad., 43 (1967), 953-956.

25. H. Ribeiro, Sur les espaces á metrique faible, Portugal. Math., 4 (1943), 21-40 and 65-68.

26. T. Shiraki, M-space, their generalizations and metrization theorems, Sci. Rep. Tokyo Kyoiku Daigaku, Sec. A., 11 (1971), 57-67.

27. F. Siwiec and J. Nagata, $A$ note on nets and metrization, Proc. Japan Acad., 44 (1968), 623-627.

28. Y. Tanaka, On open finite-to-one maps, Bull. Tokyo Gakugei Univ., Ser IV, 25 (1973), 1-13.

29. W. A. Wilson, On quasi-metric spaces, Amer. J. Math., 53 (1931), 675.

Received April 3, 1974.

University of Pittsburgh

BROOKLYN COLLEGE 



\section{PACIFIC JOURNAL OF MATHEMATICS}

\section{EDITORS}

RICHARD ARENS (Managing Editor) University of California

Los Angeles, California 90024

\section{R. A. Beaumont}

University of Washington Seattle, Washington 98105
J. DugundJI Department of Mathematics University of Southern Californıa Los Angeles, California 90007

D. Gilbarg aNd J. Milgram Stanford University Stanford, California 94305

\section{ASSOCIATE EDITORS}

E. F. BeCKENBACH

B. H. NeUmanN

F. Wolf

K. YoSHIDA

\section{SUPPORTING INSTITUTIONS}

UNIVERSITY OF BRITISH COLUMBIA CALIFORNIA INSTITUTE OF TECHNOLOGY UNIVERSITY OF CALIFORNIA MONTANA STATE UNIVERSITY UNIVERSITY OF NEVADA NEW MEXICO STATE UNIVERSITY OREGON STATE UNIVERSITY UNIVERSITY OF OREGON OSAKA UNIVERSITY
UNIVERSITY OF SOUTHERN CALIFORNIA STANFORD UNIVERSITY UNIVERSITY OF TOKYO UNIVERSITY OF UTAH WASHINGTON STATE UNIVERSITY UNIVERSITY OF WASHINGTON AMERICAN MATHEMATICAL SOCIETY NAVAL WEAPONS CENTER 


\section{Pacific Journal of Mathematics \\ Vol. 59, No. 1 \\ May, 1975}

Shashi Prabha Arya and M. K. Singal, More sum theorems for topological

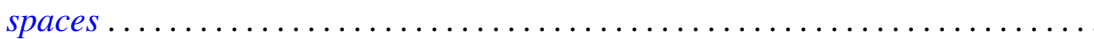

Goro Azumaya, F. Mbuntum and Kalathoor Varadarajan, On M-projective and

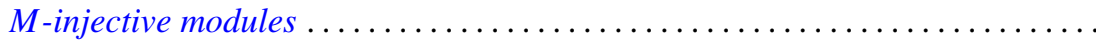

Kong Ming Chong, Spectral inequalities involving the infima and suprema of

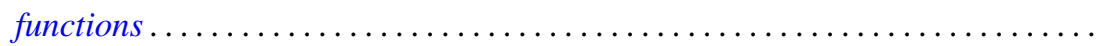

Alan Hetherington Durfee, The characteristic polynomial of the monodromy .......

Emilio Gagliardo and Clifford Alfons Kottman, Fixed points for orientation

preserving homeomorphisms of the plane which interchange two points ......

Raymond F. Gittings, Finite-to-one open maps of generalized metric spaces .......

Andrew M. W. Glass, W. Charles (Wilbur) Holland Jr. and Stephen H. McCleary,

$a^{*}$-closures of completely distributive lattice-ordered groups .............

Matthew Gould, Endomorphism and automorphism structure of direct squares of

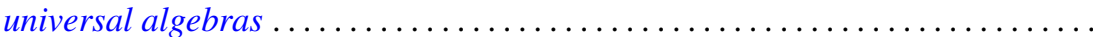

R. E. Harrell and Les Andrew Karlovitz, On tree structures in Banach spaces .....

Julien O. Hennefeld, Finding a maximal subalgebra on which the two Arens

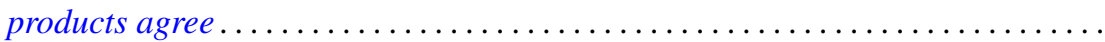

William Francis Keigher, Adjunctions and comonads in differential algebra .......

Robert Bernard Kelman, A Dirichlet-Jordan theorem for dual trigonometric

series

Allan Morton Krall, Stieltjes differential-boundary operators. III. Multivalued operators-linear relations...

Hui-Hsiung Kuo, On Gross differentiation on Banach spaces .

Tom Louton, A theorem on simultaneous observability ...

Kenneth Mandelberg, Amitsur cohomology for certain extensions of rings of algebraic integers.

Coy Lewis May, Automorphisms of compact Klein surfaces with boundary . . .

Peter A. McCoy, Generalized axisymmetric elliptic functions .

211

Muril Lynn Robertson, Concerning Siu's method for solving $y^{\prime}(t)=F(t$, $y(g(t)))$. .

Richard Lewis Roth, On restricting irreducible characters to normal

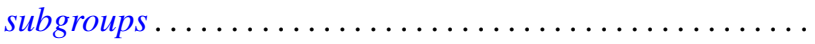

Albert Oscar Shar, $P$-primary decomposition of maps into an $H$-space .

Kenneth Barry Stolarsky, The sum of the distances to certain pointsets on the unit circle.

Bert Alan Taylor, Components of zero sets of analytic functions in $C^{2}$ in the unit ball or polydisc

Michel Valadier, Convex integrands on Souslin locally convex spaces ...

Januario Varela, Fields of automorphisms and derivations of $C$

Arnold Lewis Villone, A class of symmetric differential operators with deficiency

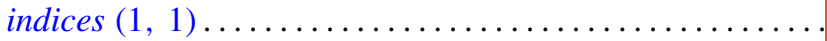

